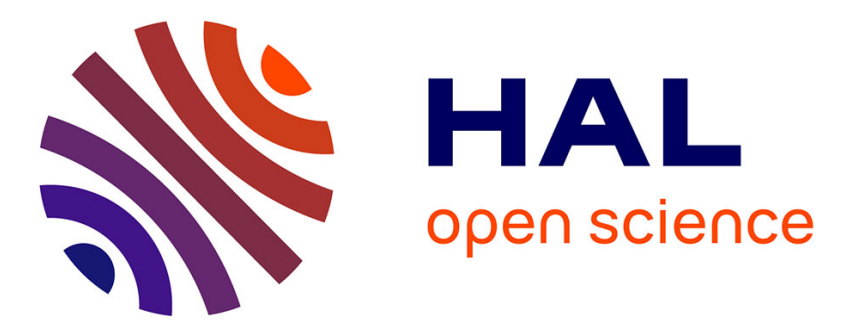

\title{
A Variable-Length Cell Road Traffic Model: Application to Ring Road Speed Limit Optimization
}

\author{
Carlos Canudas de Wit, Antonella Ferrara
}

\section{To cite this version:}

Carlos Canudas de Wit, Antonella Ferrara. A Variable-Length Cell Road Traffic Model: Application to Ring Road Speed Limit Optimization. CDC 2016 - 55th IEEE Conference on Decision and Control, Dec 2016, Las Vegas, NV, United States. hal-01391239

\section{HAL Id: hal-01391239 \\ https://hal.science/hal-01391239}

Submitted on 3 Nov 2016

HAL is a multi-disciplinary open access archive for the deposit and dissemination of scientific research documents, whether they are published or not. The documents may come from teaching and research institutions in France or abroad, or from public or private research centers.
L'archive ouverte pluridisciplinaire HAL, est destinée au dépôt et à la diffusion de documents scientifiques de niveau recherche, publiés ou non, émanant des établissements d'enseignement et de recherche français ou étrangers, des laboratoires publics ou privés. 


\title{
A Variable-Length Cell Road Traffic Model: Application to Ring Road Speed Limit Optimization
}

\author{
Carlos Canudas-de-Wit* and Antonella Ferrara ${ }^{\diamond}$
}

\begin{abstract}
In this paper we propose a variable speed control strategy based on a new Variable-Length cell transmission Model (VLM). The VLM differs from the standard Cell Transmission model in that only a limited number of (variable length) cells are used. Road network is subdivided into several sections which are assumed to be composed of a downstream congested cell followed by a free upstream cell. Both cells have variable lengths and are described by two lumped densities (one congested, the other free). One more state describing the length variation completes the model for each section. The paper also introduces an associated optimal speed control design based on the proposed VLM. The method is illustrated on a closed ring road and is shown to optimize the traveling time per turn.
\end{abstract}

\section{INTRODUCTION}

Modern technologies are now a well recognized vector to alleviate traffic congestions problems. First steep is to equip the roads with sensors collecting [4] and processing data [20], in order to decide optimal traffic control policies based on reliable traffic models [13], [15], [14], [17], [18], [8], [9]. Physicists, mathematicians and engineers have developed models of vehicular traffic for almost fifty years [21], but models useful for control design are still a limited number. Control designers have preference for simple macroscopic models capturing fundamental physical features like vehicle conservation and shock wave propagation, often inspired by analogies from flows dynamics in fluids or gases.

For instance, the well-known Daganzo's Cell Transmission Model (CTM) [5] comes from a discretization into cells (corresponding to small portions of the road) of the LWR continuum model proposed by Lighthill and Whitham [16], and Richards [19]. The CTM keeps track of the number of vehicles in each cell, and at every time step determines the number of vehicles that cross the boundaries between adjacent cells. The interface flows between cells can be captured by the well-known and compact demand/supply formulation (see [12] for a complete study of CTM major mathematical properties).

One limitation of the CTM model is due to its complexity which is determined by the number of cells into which the considered road stretch is subdivided. Using the CTM

* Carlos Canudas-de-Wit. CNRS, Department of Automatic Control, GIPSA-lab, Grenoble, France.

$\diamond$ Antonella Ferrara, Dipartimento di Ingegneria Industriale e dell'Informazione, University of Pavia, Italy to model large scale traffic networks results in a highdimensional switched linear model with exponential complexity.

The previous considerations have been the motivations for searching new low-dimensional macroscopic models allowing tractable control design with reduced complexity. Among those there is the so-called Link-Transmission model (LTM) which is basically a CTM with a single cell, but with the limitation that the shock propagation is only possible between links. This implies that the shock waves propagation inside the link is not captured, while it is captured by the CTM does (see [22] for further discussion). The use of this model is often limited to the context of traffic light control in urban network.

Store and forward models are some sort of queue models integrating the difference between the total inflows and outflows. The model traces back to the original works of Gazis and Potts (1963) [10] and has since then been used in various road traffic control optimization studies [1]. Those models integrate several simplifications at the cost of the loss of many of the model kinematic original properties. Stochastic version of this models have been also considered[23]. A common observed limitation is that queues are "vertical" with unlimited capacity. Therefore, it does not permit saturation of a downstream link that blocks the movement of vehicles from an upstream link. This may result in motion-blocking problems as observed by [23], [2], and in a substantial deviation from waves propagations as predicted from the original LWR model.

In this paper, we propose a new aggregated VariableLength cell transmission Model (VLM) aiming also at complexity reduction by limiting the number of states per link, which stems from the ground base ideas presented in [3]. In this paper we present an improved model with respect to that in [3], showing in particular that its derivation results from averaging the original LWR model while ensuring that the wave propagations are suitable considered, not only at the level of the link but also between neighbor links. Essentially, the model consists of three lumped state variables per link one of which explicitly describes the position of the congestion wave front (or the queue length). In this paper we also formally address the most prominent mathematical model properties including; the consistency with respect to its inherent mass (or vehicle) conservation law, and the 
capability of accurately describing the propagation of shock and advection waves. We also equipped the model with small fixed boundary cells to avoid singularities when the link is either fully congested or fully free.

The proposed model can be satisfactorily used in several possible scenarios. Moreover, it possesses a sufficient level of accuracy and the appropriate simplicity to be adopted as a basis to design controllers for traffic systems. This is testified by its effective use in the context of optimal speed design illustrated in [6], [7]. Even in the present paper, to better emphasize the control design oriented nature of the VSL model, we present the model utilization in a closed ring road, and the possibility of exploiting such a model to optimize the speed limits, so as to improve the traveling time in that scenario.

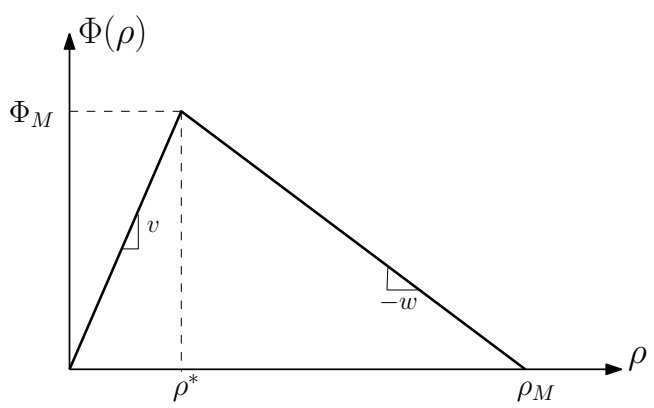

Fig. 1. Schematic representation of the triangular fundamental diagram.

\section{Preliminaries: Conservation laWs And CTM}

The simplest continuous macroscopic traffic model is the so-called LWR model, proposed by Lighthill, Whitham [16], and Richards [19]. It is based on the vehicle conservation's principle, and on the assumption that the traffic can be described by the empiric relation between flow, $\varphi$, and density, $\rho$, as: $\varphi=\Phi(\rho)$, where the function $\Phi(\cdot)$ is called Fundamental Diagram (see Fig. 1), which can be defined in its simplest form by a triangle given by

$$
\Phi(\rho)=\min \left\{v \rho,-w\left(\rho-\rho_{M}\right)\right\}
$$

where $v$ is the maximum velocity at free-flow, and $-w$ defines the speed at which congestion travels upstream. $\Phi(\rho)$ has its maximum at $\Phi_{M}=\Phi\left(\rho^{*}\right)$ describing the maximum capacity of the road. The critical density $\rho^{*}$ defines the boundary between the decongested and the congested modes, while $\rho_{M}$ is the maximum density that the road can withstand.

The evolution of the number of vehicles, $N$, within any spatial section $(0, L)$ is given by the conservation law

$$
\frac{\mathrm{d}}{\mathrm{d} t} N=\varphi_{\text {in }}-\varphi_{\text {out }}, \quad N=\int_{0}^{L} \rho(x, t) \mathrm{d} x
$$

where $\varphi_{\text {in }}$ and $\varphi_{\text {out }}$ are the input (at $x=L$ ) and output (at $x=0$ ) flows at the boundaries of the road section of length
L. Equation (1) can be rewritten (see [16]) as a hyperbolic equation involving only the density

$$
\partial_{t} \rho+\partial_{x} \Phi(\rho)=\partial_{t} \rho+\partial_{\rho} \Phi \cdot \partial_{x} \rho=0
$$

The macroscopic continuous density dynamics is then given by the LWR Cauchy problem described by (2) with the initial condition $\rho(x, 0)=\rho^{0}(x)$.

Assume now that the road section is subdivided into $n$-cells of constant length $\Delta l_{i}$. Let us denote with $\rho_{i}$ the density of the $i^{t h}$ cell of the section. Then, the number of vehicles per cell can be computed as $N_{i}=\rho_{i} \Delta l_{i}$.

A well-known efficient first-order numerical method to integrate (2) is the Godunov scheme [11]. This process leads to discrete space-time equations named the Cell Transmission Model (CTM) originally proposed by Daganzo in [5], which in its continuous-time version takes the following form

$$
\dot{\rho}_{i}(t)=\frac{1}{\Delta l_{i}}\left(\varphi_{i-1}(t)-\varphi_{i}(t)\right)
$$

with $\varphi_{0}=\varphi_{\text {in }}(t), \varphi_{n}=\varphi_{\text {out }}(t), \varphi_{i}$ being the interface flow between the cells $i$ and $i+1$ given as

$$
\varphi_{i}=\min \left\{D_{i}, S_{i+1}\right\}
$$

with

$$
\begin{aligned}
D_{i} & =\min \left\{v_{i} \rho_{i}, \varphi_{M, i}\right\}, \\
S_{i+1} & =\min \left\{\varphi_{M, i+1}, w_{i+1}\left(\rho_{M, i+1}-\rho_{i+1}\right)\right\}
\end{aligned}
$$

where the demand $D_{i}$ is the flow that can be delivered by the cell $i$ to the cell $i+1$, while the supply $S_{i+1}$ is the flow that can be received by the cell $i+1$ from the cell $i ; \varphi_{M, i}$ is the maximum flow allowed by the capacity of cell $i, \rho_{M, i}$ is the jam density (i.e. the maximum density that can be reached), $v_{i}$ corresponds to the free flow speed and $w_{i}$ is the congestion wave speed in cell $i$.

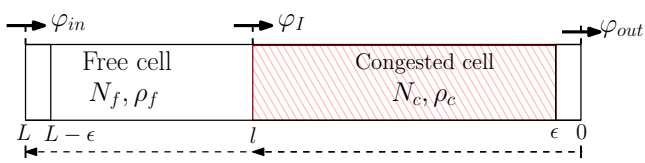

Fig. 2. Schematic diagram of the two-cell variable-length model VLM with boundaries cells introduced to avoid singularities at $l=0$, and $l=L$.

\section{NEW MACROSCOPIC MODEL WITH VARIABLE-LENGTH CELLS}

Consider a simple road section described by two cells of variable length: a downstream congested cell, of length $l$, and an upstream free cell, of length $L-l$, where $L$ is the total length of the section. The former cell is characterized by the lumped (or averaged) "congested density" $\rho_{c}$, the latter by the lumped (or averaged) "free density" $\rho_{f}$. Note that $l=l(t)$ is time-varying and specifies the position of the congestion wave front, see Fig. 2 (with $\epsilon=0$ ). Then, in contrast to the $n$-fixed cells model (3), the new macroscopic traffic model introduced in this paper, named Variable-Length cell Model (VLM), consists only of three lumped state variables, i.e. $\rho_{f}$, $\rho_{c}$ and $l$. 


\section{A. Conservation law for the variable-length cells}

Consider for consistency with the grow direction of $l$ shown in Fig. 2, that the $x$-axis is oriented with $l$ from right (downstream) to left (upstream). Therefore, the conservation law (2) now can be written as $\partial_{t} \rho=\partial_{x} \Phi(\rho)$, where we have replaced $\partial_{x}$ with $-\partial_{x}$. Moreover, let $s(t)$ be the continuous variable describing the time-varying shock wave position along the $x$ axis. Relying on $s(t)$, the following lumped (averaged) densities variables $\rho_{f}$ and $\rho_{c}$ are defined:

$$
\begin{aligned}
\rho_{f} & =\frac{1}{L-s} \int_{s}^{L} \rho(x, t) d x \\
\rho_{c} & =\frac{1}{s} \int_{0}^{s} \rho(x, t) d x
\end{aligned}
$$

Note that the density distribution is discontinuous at the boundary $x=s$. Its left, $\rho^{-}$, and right, $\rho^{+}$, limits can be written as

$$
\rho^{-}=\rho^{-}(t)=\lim _{x \rightarrow s^{-}} \rho(x, t), \quad \rho^{+}=\rho^{+}(t)=\lim _{x \rightarrow s^{+}} \rho(x, t)
$$

The time-derivatives of the lumped variables are given by

$$
\begin{aligned}
\dot{\rho}_{f} & =\frac{1}{L-s}\left[\int_{s}^{L} \partial_{t} \rho(x, t) d x-\rho^{-} \dot{s}\right]+\frac{\dot{s}}{(L-s)^{2}} \int_{s}^{L} \rho(x, t) d x \\
\dot{\rho}_{c} & =\frac{1}{s}\left[\int_{0}^{s} \partial_{t} \rho(x, t) d x+\rho^{+} \dot{s}\right]-\frac{\dot{s}}{s^{2}} \int_{0}^{s} \rho(x, t) d x
\end{aligned}
$$

Using $\partial_{t} \rho(x, t)=\partial_{x} \Phi(\rho)$ in the previous integrals, and the definitions $(5 \mathrm{a})-(5 \mathrm{~b}), \dot{\rho}_{f}$ rewrites as

$$
\begin{aligned}
\dot{\rho}_{f} & =\frac{1}{L-s}\left[\Phi(\rho(L))-\Phi\left(\rho^{-}\right)-\rho^{-} \dot{s}\right]+\frac{\dot{s}}{(L-s)} \rho_{f} \\
& =\frac{1}{L-s}\left[\varphi_{i n}-\Phi\left(\rho^{-}\right)-\rho^{-} \dot{s}\right]+\frac{\dot{s}}{(L-s)} \rho_{f}
\end{aligned}
$$

with the boundary upstream flow $\Phi(\rho(L))=\varphi_{i n}$. Similarly, we get for $\dot{\rho}_{c}$

$$
\begin{aligned}
\dot{\rho}_{c} & =\frac{1}{s}\left[\Phi\left(\rho^{+}\right)-\Phi(\rho(0))+\rho^{+} \dot{s}\right]-\frac{\dot{s}}{s} \rho_{c} \\
& =\frac{1}{s}\left[\Phi\left(\rho^{+}\right)-\varphi_{\text {out }}+\rho^{+} \dot{s}\right]-\frac{\dot{s}}{s} \rho_{c}
\end{aligned}
$$

where $\Phi(\rho(0))=\varphi_{\text {out }}$, and the boundary densities and flows at the shock wave front are denoted as $\rho^{-}, \rho^{+}$, and $\Phi\left(\rho^{+}\right), \Phi\left(\rho^{-}\right)$, respectively. From the Rankine-Hugoniot jump condition, the shock wave speed, $\dot{s}$ can be written, in the $l$ coordinates, as

$$
\dot{s}=\frac{\Phi\left(\rho^{-}\right)-\Phi\left(\rho^{+}\right)}{\rho^{+}-\rho^{-}}
$$

implying that $\Phi\left(\rho^{-}\right)+\rho^{-} \dot{s}=\Phi\left(\rho^{+}\right)+\rho^{+} \dot{s}$. The balance between the first term (left-flow) and the second term (outflow) is a direct consequence of the principle of vehicle conservation at the interface. The interface flow $\varphi_{I}$ in the moving shock wave frame is given by

$$
\varphi_{I}=\Phi\left(\rho^{+}\right)+\rho^{+} \dot{s}=\Phi\left(\rho^{-}\right)+\rho^{-} \dot{s}
$$

As such, equations (6) and (7) can be rewitten as

$$
\begin{aligned}
\dot{\rho}_{f} & =\frac{1}{L-s}\left[\varphi_{\text {in }}-\varphi_{I}+\dot{s} \rho_{f}\right] \\
\dot{\rho}_{c} & =\frac{1}{s}\left[\varphi_{I}-\varphi_{\text {out }}-\dot{s} \rho_{c}\right]
\end{aligned}
$$

Note all the variables in the previous equations, except for $s$, are expressed in the lumped form. In order to arrive at a description which only depends on lumped variables, an approximation of the shock wave position $s(t)$, denoted with $l(t)$, is introduced. The time evolution of $l(t) \in(0, L)$ is proposed to be

$$
i=\frac{\Phi\left(\rho_{f}\right)-\Phi\left(\rho_{c}\right)}{\rho_{c}-\rho_{f}}
$$

which can be regarded as the lumped version of (8). More specifically, in (12) we assume that $\Phi\left(\rho^{-}\right)$and $\Phi\left(\rho^{+}\right)$ are approximated by $\Phi\left(\rho_{f}\right)$ and $\Phi\left(\rho_{c}\right)$, respectively. This approximation makes sense as the densities in each of the variable cells are assumed to be scalar and constant over the space variables, and not distributions.

Then on the basis of (12), equations (10) and (11) can be rewritten as

$$
\begin{aligned}
\dot{\rho}_{f} & =\frac{1}{L-l}\left[\varphi_{\text {in }}-\varphi_{I}+i \rho_{f}\right] \\
\dot{\rho}_{c} & =\frac{1}{l}\left[\varphi_{I}-\varphi_{\text {out }}-i \rho_{c}\right]
\end{aligned}
$$

\section{B. Full dynamics}

Note that the lumped model provides a piece-wise value of the traffic density for each of the two portions (the free and the congested one) of the road section, in opposition to the spatially distributed formulation. The boundary density values in the lumped case are then given as

$$
\rho^{+}=\rho_{c}, \quad \text { and } \quad \rho^{-}=\rho_{f}
$$

so that equation (9) writes now as

$$
\varphi_{I}=\Phi\left(\rho_{c}\right)+\rho_{c} i=\Phi\left(\rho_{f}\right)+\rho_{f} i
$$

from which we get

$$
-\varphi_{I}+\rho_{f} \dot{l}=-\Phi\left(\rho_{f}\right) \quad \text { and } \quad \varphi_{I}-\rho_{c} i=\Phi\left(\rho_{c}\right)
$$

Substitution of these expression into (13) and (14), together with (12), gives

$$
\begin{aligned}
\dot{\rho}_{f} & =\frac{1}{L-l}\left[\varphi_{\text {in }}-\Phi\left(\rho_{f}\right)\right] \\
\dot{\rho}_{c} & =\frac{1}{l}\left[\Phi\left(\rho_{c}\right)-\varphi_{\text {out }}\right] \\
\dot{l} & =\frac{\Phi\left(\rho_{f}\right)-\Phi\left(\rho_{c}\right)}{\rho_{c}-\rho_{f}}
\end{aligned}
$$

Equations (17)-(19) define the new macroscopic traffic model we propose. This model is a lumped variables model based on three state variables only. As for its structure, it is simpler than classical continuous macroscopic models, as well as of $n$-fixed cells models. Nevertheless, as discussed in 
the next section, it features interesting properties which allow it to capture the relevant phenomena of traffic dynamics.

Remark 1 The VLM owns fundamental mathematical properties consistent with the constructive assumptions. For instance, it can be shown that densities $\rho_{c}$, and $\rho_{f}$ remain in their definition domain $\Omega_{f}=\left\{\rho: \rho \in\left[0, \rho^{*}\right]\right\}$, and $\Omega_{c}=\left\{\rho: \rho \in\left[\rho^{*}, \rho_{M}\right]\right\}$ as long the density initial conditions are taking values inside the respective domains. However, to ensure that $l \in \Omega_{l}$, where $\Omega_{l}=\{l: l \in(0, L)\}$, additional mechanisms need to be implemented to treat the singular points where $l$ is equal to either 0 or $L$.

\section{Special cases of singular points}

The VLM model presents three degenerate cases when $l=$ $0, l=L$, and $\rho_{f}=\rho_{c}$, respectively. This subsection describes how the original model can be reformulated to cope with such cases.

1) Singular points for $l(t)$ : Consider first the case where the variable length $l(t)$ approaches one of the two possible boundary values. In those cases, the idea is to create a $\varepsilon$ boundary layer at the two extremes of the considered road section and stop the evolution of $l(t)$ when it reaches the $\varepsilon$ boundary layer borders. When this happens, the model collapses into the standard CTM model with fixed length cells, and the density variables evolve according to the conventional "Demand-Supply" mechanism, with the standard notation

$$
D(\rho)=\min \left\{v \rho, \varphi_{M}\right\}, \quad S(\rho)=\min \left\{-w\left(\rho-\rho_{M}\right), \varphi_{M}\right\}
$$

This idea is sketched in Fig. 2.

Let us consider an arbitrarily small $\varepsilon$ such that $0<\varepsilon \ll L$. Then the following two conditions can be introduced:

$$
\begin{aligned}
\mathcal{C}_{0} & =\left\{(l=\varepsilon) \cap\left(D\left(\rho_{f}\right) \leq S\left(\rho_{c}\right)\right)\right\} \\
\mathcal{C}_{L} & =\left\{(l=L-\varepsilon) \cap\left(D\left(\rho_{f}\right) \geq S\left(\rho_{c}\right)\right)\right\}
\end{aligned}
$$

Condition $\mathcal{C}_{0}$ implies that the lower extreme is reached while the congestion front wave is moving downstream. Symmetrically, condition $\mathcal{C}_{L}$ indicates that the upper extreme is reached while the congestion front wave is moving upstream. For those specific cases, the following model modification is proposed:

- If $\mathcal{C}_{0}$ holds, the interface flow is $\varphi_{I}=$ $\min \left\{D\left(\rho_{f}\right), S\left(\rho_{c}\right)\right\}=D\left(\rho_{f}\right)$, and then the model becomes

$$
\begin{aligned}
\dot{\rho}_{f} & =\frac{1}{L-\varepsilon}\left[\varphi_{\text {in }}-D\left(\rho_{f}\right)\right] \\
\dot{\rho}_{c} & =\frac{1}{\varepsilon}\left[D\left(\rho_{f}\right)-\varphi_{\text {out }}\right] \\
\dot{l} & =0
\end{aligned}
$$

Note that in this case, the variation domain of the state variables is partially modified. The congested density variable can now take values smaller than the critical density $\rho^{*}$, i.e. $\rho_{c} \in\left[0, \rho_{M}\right]$, while the free density variable remains in its original domain, i.e. $\rho_{f} \in \Omega_{f}$.
- If $\mathcal{C}_{L}$ holds, the interface flow is $\varphi_{I}=$ $\min \left\{D\left(\rho_{f}\right), S\left(\rho_{c}\right)\right\}=S\left(\rho_{c}\right)$, and then the model becomes

$$
\begin{aligned}
\dot{\rho}_{f} & =\frac{1}{\varepsilon}\left[\varphi_{\text {in }}-S\left(\rho_{c}\right)\right] \\
\dot{\rho}_{c} & =\frac{1}{L-\varepsilon}\left[S\left(\rho_{c}\right)-\varphi_{\text {out }}\right] \\
\dot{l} & =0
\end{aligned}
$$

In this case, the free density variable can take values larger than the critical density $\rho^{*}$, i.e. $\rho_{f} \in\left[0, \rho_{M}\right]$, while the congested density variable remains in its original domain, i.e. $\rho_{c} \in \Omega_{c}$.

Now consider the third singular point, i.e. $\rho_{f}=\rho_{c}$. From the fundamental diagram in Fig. 1, it is apparent that this singularity only occurs at $\rho_{f}=\rho_{c}=\rho^{*}$, where $\Phi\left(\rho_{f}\right)=\Phi\left(\rho_{c}\right)=\varphi_{M}$. This implies that equation (19) becomes undetermined. As a consequence, in order to extend the proposed model validity to this case, a regularization mechanism needs to be introduced. To this end, let $e_{f}=$ $\rho_{f}-\rho^{*} \leq 0$ and $e_{c}=\rho_{c}-\rho^{*} \geq 0$, we have that $e_{f}=-\left|e_{f}\right|$, and $e_{c}=\left|e_{c}\right|$. Hence, (19) can be rewritten as

$$
i=\frac{-v\left|e_{f}\right|+w\left|e_{c}\right|}{\left|e_{f}\right|+\left|e_{c}\right|}
$$

Let us denote with $V\left(e_{f}, e_{c}\right)=\left|e_{f}\right|+\left|e_{c}\right|$, and with $\sigma\left(e_{f}, e_{c}\right)=\epsilon e^{-\alpha V^{2}\left(e_{f}, e_{c}\right)}, \epsilon$ and $\alpha$ being strictly positive coefficients, a suitable regularizing term. Then, the following regularization for (19)

$$
i=\frac{-v\left|e_{f}\right|+w\left|e_{c}\right|}{V\left(e_{f}, e_{c}\right)+\sigma\left(e_{f}, e_{c}\right)}, \quad \lim _{V\left(e_{f}, e_{c}\right) \rightarrow 0} i=0
$$

is proposed, which in the original system coordinates writes as

$$
i=\frac{\Phi\left(\rho_{f}\right)-\Phi\left(\rho_{c}\right)}{\rho_{c}-\rho_{f}+\sigma\left(\rho_{c}, \rho_{f}\right)}
$$

with $\sigma\left(\rho_{c}, \rho_{f}\right)=\epsilon e^{-\alpha\left(\rho_{f}-\rho_{c}\right)^{2}}$.

\section{Singularity-free full VLM}

Considering the different regularizations introduced previously, and noticing that $l=\varepsilon$, and $l=L-\varepsilon$, at $\mathcal{C}_{0}$ and $\mathcal{C}_{L}$, respectively, the full model can be compactly written as follows

$$
\begin{aligned}
\dot{\rho}_{f} & =\frac{1}{L-l}\left\{\begin{array}{lll}
\varphi_{\text {in }}-D\left(\rho_{f}\right) & \text { if } \mathcal{C}_{0} \\
\varphi_{\text {in }}-S\left(\rho_{c}\right) & \text { if } \mathcal{C}_{L} \\
\varphi_{\text {in }}-\Phi\left(\rho_{f}\right) & \text { else }
\end{array}\right. \\
\dot{\rho}_{c} & =\frac{1}{l}\left\{\begin{array}{cl}
D\left(\rho_{f}\right)-\varphi_{\text {out }} & \text { if } \mathcal{C}_{0} \\
S\left(\rho_{c}\right)-\varphi_{\text {out }} & \text { if } \mathcal{C}_{L} \\
\Phi\left(\rho_{c}\right)-\varphi_{\text {out }} & \text { else }
\end{array}\right. \\
\dot{l} & =\left\{\begin{array}{cc}
0 & \text { if } \mathcal{C}_{0} \cup \mathcal{C}_{L} \\
\frac{\Phi\left(\rho_{f}\right)-\Phi\left(\rho_{c}\right)}{\rho_{c}-\rho_{f}+\sigma\left(\rho_{c}, \rho_{f}\right)} & \text { else }
\end{array}\right.
\end{aligned}
$$




\section{RAREFACTION WAVES IN THE VLM}

Model (29)-(31) has been designed under the implicit assumption of the existence of a bottleneck originating an important change of capacity at the boundary $l=0$, and producing a congestion whose origin in the space remains fixed. However, there are other situations in which both spatial boundaries of the congestion may evolve in time. This is the case when a sudden change from the congested mode to the free mode occurs at the downstream boundary (i.e. because of the traffic light switching from red to green, or of a car accident being eventually resolved) producing a rarefaction (or fan) wave at the downstream boundary.

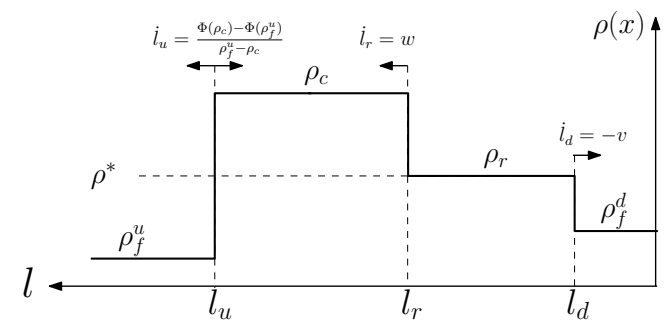

Fig. 3. Illustration of the variables involved in the model including rarefaction waves.

Consider the variables as defined in Fig. 3, with $\rho_{f}^{u}, \rho_{c}, \rho_{r}, \rho_{f}^{d}$ being the densities, and $l_{u}, l_{r}, l_{d}$ being the associated cell lengths subject to the following constraints: $L>l_{u} \geq l_{r} \geq l_{d}>0$, and $0<\rho_{f}^{d}<\rho^{*}<\rho_{c}<\rho_{M}$. The left and the right boundary of the rarefaction wave are indicated by $l_{r}, l_{d}$ respectively. Assuming the rarefaction wave is initiated at $l=l_{0}$, the solution of the Riemann problem with initial left and right distributions $\rho^{-}=\rho_{c}$, and $\rho^{+}=\rho_{f}^{d}$ is:

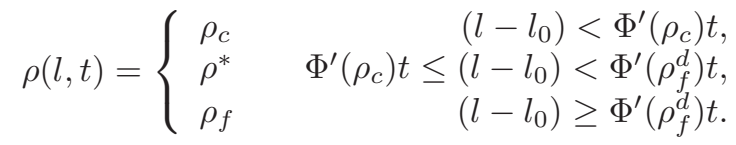

For the triangular piece-wise fundamental diagram, we have $\Phi^{\prime}\left(\rho_{c}\right)=w$, and $\Phi^{\prime}\left(\rho_{f}^{d}\right)=-v$ (in the $l$-direction). We have then the formation of two shock waves: one traveling at velocity $w$, and another traveling at velocity $-v$, with critical density $\rho_{r}=\rho^{*}$ as the intermediate state.

The model can now be constructed by following the same ideas on vehicles conservation laws used previously, i.e.

$$
\begin{aligned}
\dot{N}_{f}^{u} & =\varphi_{\text {in }}-\varphi_{I}^{u} \\
\dot{N}_{c} & =\varphi_{I}^{u}-\varphi_{I}^{r} \\
\dot{N}_{r} & =\varphi_{I}^{r}-\varphi_{I}^{d} \\
\dot{N}_{f}^{d} & =\varphi_{I}^{d}-\varphi_{\text {out }}
\end{aligned}
$$

with $N_{f}^{u}=\left(L-l_{u}\right) \rho_{f}^{u}, N_{c}=\left(l_{u}-l_{r}\right) \rho_{c}, N_{r}=\left(l_{r}-L_{d}\right) \rho_{r}$, $N_{f}^{d}=l_{d} \rho_{f}^{d}$, and $\varphi_{I}^{u}, \varphi_{I}^{r}, \varphi_{I}^{d}$ being the interface flows at $l_{u}, l_{r}, l_{d}$, respectively. As before, each interface flow can be computed using the speed function $i_{-/+}=f\left(\rho^{-}, \rho^{+}\right)=$ $\frac{\Phi\left(\rho^{+}\right)-\Phi\left(\rho^{-}\right)}{\rho^{-}-\rho^{+}}$of each interface, and its corresponding left and right densities $\rho^{-}, \rho^{+}$. This gives: $\varphi_{I}^{-/+}=\rho^{-} i_{-/+}+$ $\Phi\left(\rho^{-}\right)=\rho^{+} i_{-l_{+}+} \Phi\left(\rho^{+}\right)$. The full model is now given by

$$
\begin{aligned}
\dot{\rho}_{f}^{u} & =\frac{1}{L-l_{u}}\left[\varphi_{\text {in }}-\Phi\left(\rho_{f}^{u}\right)\right] \\
\dot{\rho}_{c} & =0 \\
\dot{\rho}_{r} & =0 \\
\dot{\rho}_{f}^{d} & =\frac{1}{l_{d}}\left[\Phi\left(\rho_{f}^{d}\right)-\varphi_{\text {out }}\right]
\end{aligned}
$$

and

$$
\begin{aligned}
& i_{u}=\left\{\begin{array}{lll}
f\left(\rho_{f}^{u}, \rho_{c}\right) & \text { if } \quad l_{u}>l_{r} \\
f\left(\rho_{f}^{u}, \rho_{r}\right)=-v & \text { if } \quad l_{u}=l_{r}
\end{array}\right. \\
& i_{r}=\left\{\begin{array}{lll}
f\left(\rho_{c}, \rho_{r}\right)=w & \text { if } \quad l_{u}>l_{r} \\
f\left(\rho_{f}^{u}, \rho_{r}\right)=-v & \text { if } \quad l_{u}=l_{r}
\end{array}\right. \\
& \dot{l}_{d}=f\left(\rho_{r}, \rho_{f}^{d}\right)=-v \quad \text { if } \quad l_{r} \geq l_{d}
\end{aligned}
$$

with initial conditions $\rho_{f}^{u}(0), \rho_{c}(0), \rho_{r}(0)=\rho^{*}, \rho_{f}^{d}(0)$, and $l_{f}^{u}(0)>l_{r}(0)=l_{f}^{d}(0)=l_{0}$.

Remark 2 Because of the particular initial density distribution, and of equations (38)-(39), both densities $\rho_{c}(t)=\rho_{c}(0)$, and $\rho_{r}(t)=\rho^{*}$ remain constant. The rarefaction wave reaches in finite time the upstream free state $\left(i . e . l_{u}=l_{r}\right)$, giving raise to a new shock wave whose values are below the critical density and moves to the right with velocity $v$, i.e. $\dot{l}_{u}=\dot{l}_{r}=-v$.

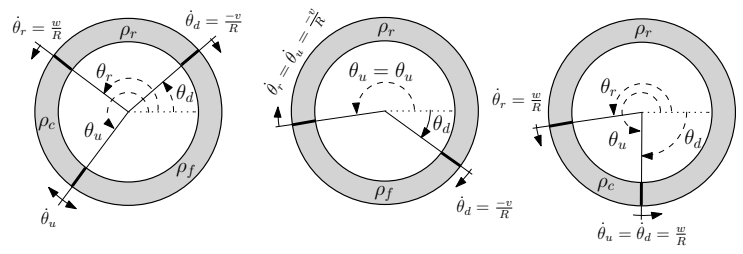

Fig. 4. Illustration of the ring-road scenario. Left figure shown the scenario with the associated variables. Center (class A) and right (class B) figures shown the two possible equilibria classes.

\section{VARIABle SPEED OPTIMIZATION IN A CLOSED RING ROAD}

In this section, a case study of a closed ring-road scenario shown in Fig. 4 is considered to illustrate the main model properties and how the model can be used for variable speed optimization.

\section{A. Ring-road Variable Length model}

Let $l_{u}=R \theta_{u}, l_{r}=R \theta_{r}, l_{d}=R \theta_{d}, \rho_{f}^{u}=\rho_{f}^{d}=\rho_{f}$, $\varphi_{\text {in }}=\varphi_{\text {out }}$. Model (37)-(43), in the set $\Omega_{\theta}=$ $\left\{\left(\theta_{u}, \theta_{r}, \theta_{d}\right): \theta_{u} \geq \theta_{r} \geq \theta_{d}\right\} \backslash\left\{\left(\theta_{u}, \theta_{r}, \theta_{d}\right): \theta_{u}=\theta_{r}=\theta_{d}\right\}$, 
can be written in the circular coordinates as,

$$
\begin{aligned}
\dot{\rho}_{f} & =0, \quad \rho_{f}(0)=\rho_{f}^{0} \\
\dot{\rho}_{c} & =0, \quad \rho_{c}(0)=\rho_{c}^{0} \\
\dot{\rho}_{r} & =0, \quad \rho_{r}(0)=\rho^{*} \\
\dot{\theta}_{u}=\frac{\dot{l}_{u}}{R} & =\frac{1}{R} \begin{cases}f\left(\rho_{f}, \rho_{c}\right) & \text { if } \theta_{u}>\theta_{r} \\
f\left(\rho_{f}, \rho_{r}\right)=-v & \text { if } \theta_{u}=\theta_{r}\end{cases} \\
\dot{\theta}_{r}=\frac{i_{r}}{R} & =\frac{1}{R}\left\{\begin{array}{lll}
f\left(\rho_{c}, \rho_{r}\right)=w & \text { if } \theta_{u}>\theta_{r} \\
f\left(\rho_{f}, \rho_{r}\right)=-v & \text { if } \theta_{u}=\theta_{r}
\end{array}\right. \\
\dot{\theta}_{d}=\frac{i_{d}}{R} & =\frac{1}{R}\left\{\begin{array}{lll}
f\left(\rho_{r}, \rho_{f}\right)=-v & \text { if } \theta_{r} \geq \theta_{d} \\
f\left(\rho_{r}, \rho_{c}\right)=w & \text { if } \theta_{d}=\theta_{u}
\end{array}\right.
\end{aligned}
$$

with initial conditions $\left(\theta_{u}^{0}, \theta_{r}^{0}, \theta_{d}^{0}\right) \in \Omega_{\theta}$.

\section{B. Equilibrium points of the VLM of the ring road}

The model (44)-(49) has in general two classes of equilibrium points depending on the initial conditions. One class (Class A) is composed of one part in free-flow and the other at the critical density, while the second class (Class B) consists of one part in congested mode, and the other at the critical density, see Fig. 4.

Consider the following definitions:

$$
\begin{aligned}
\Delta \theta_{f}(t) & =2 \pi+\theta_{d}(t)-\theta_{u}(t) \\
\Delta \theta_{c}(t) & =\theta_{u}(t)-\theta_{r}(t) \\
\Delta \theta_{*}(t) & =\theta_{r}(t)-\theta_{d}(t)
\end{aligned}
$$

with the notation $\Delta \theta_{(\cdot)}(0)=\Delta \theta_{(\cdot)}^{0}$.

Lemma 1 Consider the ring-road model (44)-(49), with initial conditions $\theta_{u}^{0}>\theta_{r}^{0} \geq \theta_{d}^{0}$. Let

$$
\begin{gathered}
a=\frac{\rho_{f}^{0}}{\rho_{c}^{0}-\rho_{f}^{0}}, \quad b=\frac{\Phi\left(\rho_{c}^{0}\right)}{\rho_{c}^{0}-\rho_{f}^{0}}, \quad \beta=\frac{\Delta \theta_{f}^{0}}{\Delta \theta_{c}^{0}} \\
c(\beta, a, b)=\frac{b(1+\beta)+\beta w}{a(1+\beta)+1}, \quad f_{0}=f\left(\rho_{f}^{0}, \rho_{c}^{0}\right)
\end{gathered}
$$

Then:

A) The system converges in a finite time $t_{1}=R \frac{\Delta \theta_{c}^{0}}{w-f_{0}}$, to the Class-A equilibrium points if initial conditions satisfy:

$$
\frac{f_{0}+v}{w-f_{0}}<\beta
$$

with the final angles given as:

$$
\begin{aligned}
& \Delta \theta_{c}\left(t_{1}\right)=0 \\
& \Delta \theta_{f}\left(t_{1}\right)=\Delta \theta_{f}^{0}-\frac{v+f_{0}}{w-f_{0}} \Delta \theta_{c}^{0} \\
& \Delta \theta_{*}\left(t_{1}\right)=\Delta \theta_{*}^{0}+\frac{v+w}{w-f_{0}} \Delta \theta_{c}^{0}
\end{aligned}
$$

with $\Delta \theta_{f}\left(t_{1}\right)+\Delta \theta_{*}\left(t_{1}\right)=2 \pi$.

$B)$ The system converges in a finite time $t_{2}=R \frac{\Delta \theta_{f}^{0}}{v+f_{0}}$, to the Class- $B$ equilibrium points if initial conditions satisfy:

$$
\frac{f_{0}+v}{w-f_{0}}>\beta
$$

with the final angles given as:

$$
\begin{aligned}
& \Delta \theta_{c}\left(t_{2}\right)=\Delta \theta_{c}^{0}-\frac{w-f_{0}}{v+f_{0}} \Delta \theta_{f}^{0}, \\
& \Delta \theta_{f}\left(t_{2}\right)=0, \\
& \Delta \theta_{*}\left(t_{2}\right)=\Delta \theta_{*}^{0}+\frac{v+w}{v+f_{0}} \Delta \theta_{f}^{0}
\end{aligned}
$$

with $\Delta \theta_{c}\left(t_{2}\right)+\Delta \theta_{*}\left(t_{2}\right)=2 \pi$.

Proof. The proof is straightforward by computing the angle evolutions of the solutions

$$
\begin{aligned}
& \theta_{r}(t)=\theta_{r}^{0}+\frac{w}{R} t \\
& \theta_{u}(t)=\theta_{u}^{0}+\frac{f_{0}}{R} t \\
& \theta_{d}(t)=\theta_{d}^{0}-\frac{v}{R} t
\end{aligned}
$$

and noticing that $f_{0}$ can be rewritten as

$$
f_{0}=\frac{\phi\left(\rho_{f}^{0}\right)-\Phi\left(\rho_{c}^{0}\right)}{\rho_{c}^{0}-\rho_{f}^{0}}=v \frac{\rho_{f}^{0}}{\rho_{c}^{0}-\rho_{f}^{0}}-\frac{\Phi\left(\rho_{c}^{0}\right)}{\rho_{c}^{0}-\rho_{f}^{0}}=a v-b
$$

For Class $A$, we require the existence of a finite time $t_{1}$ such that: i) $\theta_{r}\left(t_{1}\right)=\theta_{u}\left(t_{1}\right)$, and $\left.i i\right) \theta_{u}\left(t_{1}\right)<2 \pi+\theta_{d}\left(t_{1}\right)$. Now, from the above solutions, we get

$$
\begin{aligned}
\theta_{r}^{0}+\frac{w}{R} t_{1} & =\theta_{u}^{0}+\frac{f_{0}}{R} t_{1} \\
\theta_{u}^{0}+\frac{f_{0}}{R} t_{1} & <2 \pi+\theta_{d}^{0}-\frac{v}{R} t_{1}
\end{aligned}
$$

Then, $t_{1}$ is obtained from the first equation. The velocity range comes from the second equations where we replace $t_{1}$, and noting the fact that $f_{0}<0$, and hence $a v-b<0$.

For Class $B$, we require the existence of a finite time $t_{2}$ such that: i) $\theta_{u}\left(t_{2}\right)=2 \pi+\theta_{d}\left(t_{2}\right)$, and $\left.i i\right) \theta_{u}\left(t_{2}\right)>\theta_{r}\left(t_{2}\right)$. Now, from the above solutions, we get

$$
\begin{aligned}
& \theta_{u}^{0}+\frac{f_{0}}{R} t_{2}=2 \pi+\theta_{d}^{0}-\frac{v}{R} t_{2} \\
& \theta_{u}^{0}+\frac{f_{0}}{R} t_{2}>\theta_{r}^{0}+\frac{w}{R} t_{2}
\end{aligned}
$$

As before, $t_{2}$ is obtained from the first equation. The velocity range comes from the second equation where we replace $t_{2}$.

$$
\diamond \diamond \diamond
$$

Figure 5 shows the velocity admissible domain as a function of the initial angles defined by $\beta$. Note this domain corresponds to free-flow velocities which are consistent with the model densities definition. For instance, the lower admissible value $v_{m}=b /(a+1)$ is equal to $\Phi\left(\rho_{c}^{0}\right) / \rho_{c}^{0}$, and maximum velocity bound $v_{M}=(b+w) / a$ is equal to $-w\left(1-\rho_{M} / \rho_{f}^{0}\right)$. As long as $v \in \Omega_{v}=\left(v_{m}, v_{M}\right)$ the evolution of $\rho_{f}(t), \rho_{c}(t)$ will remain in the corresponding free and congested part of the fundamental diagram. The curve $c(\beta, a, b)$ is the velocity boundary between the two possible equilibria classes.

Note that this allows for some flexibility in the selection of $v \in \Omega_{v}$, and therefore opens the possibility to optimize 


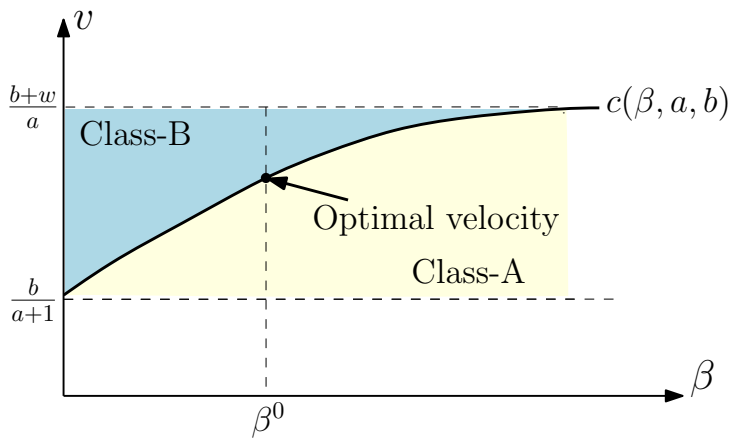

Fig. 5. Admissible velocity domain as a function of the initial conditions. The domain is limited by $v \in\left(v_{m}, v_{M}\right)$, where $v_{m}=b /(a+1), v_{M}=$ $(b+w) / a$

the network operation. For instance by minimizing the lap time, i.e.

$$
v_{o p t}=\min _{v \in \Omega_{v}, i \in 1,2} J(v, i)
$$

where

$$
J(v, i)=R\left[\frac{\Delta \theta_{*}\left(t_{i}\right)}{v}+\frac{\Delta \theta_{f}\left(t_{i}\right)}{v}+\frac{\Delta \theta_{c}\left(t_{i}\right)}{v_{m}}\right] .
$$

A particular case of interest is to find the optimal free-speed for the Class-A equilibria, which can be easily computed by observing Figure 5, that is

$$
v_{o p t}=\min _{v \in \Omega_{v}, i=1} J(v, 1)=c(\beta, a, b)
$$

leading to $J\left(v_{o p t}, 1\right)=2 \pi R / c(\beta, a, b)$.

\section{Simulation of the ring road}

The two possible cases (A and B) mentioned in Subsection V-B can be reproduced in simulation. The considered physical parameters of the model are given in table $\mathrm{V}-\mathrm{C}$. The initial conditions are $\rho_{f}^{0}=30 \mathrm{veh} / \mathrm{km}, \rho_{c}^{0}=150 \mathrm{veh} / \mathrm{km}$ for case A, and $\rho_{f}^{0}=10 \mathrm{veh} / \mathrm{km}, \rho_{c}^{0}=100 \mathrm{veh} / \mathrm{km}$ for case B. In both cases the radius is equal to $R=0.8 \mathrm{~km}$, and the initial congestion length is $l_{u}=\frac{2 \pi}{3} R=1.67 \mathrm{~km}$ (with $\left.\theta_{u}(0)=\frac{2 \pi}{3}, \operatorname{and} \theta_{r}(0)=0\right)$. Vehicles are assumed to flow in a clockwise direction. Due to these initial conditions, in case A the rotation speed is $\dot{\theta}_{u}=3.33 \mathrm{~km} / \mathrm{h}$, while in case $\mathrm{B}$ is $\dot{\theta}_{u}=-24.44 \mathrm{~km} / \mathrm{h}$. Results are shown in Fig. 6 for both cases. Six different snapshots are reported. From the figure, it is possible to observe how the rarefaction process evolves and how the equilibria are reached. As expected, two possible equilibria occur. In case $\mathrm{A}$, after that the rarefaction wave is produced, $\theta_{u}$ reaches $\theta_{r}$ and the equilibrium is given by a zone at $\rho^{*}$ and by one at $\rho_{f}^{0}$. In case $\mathrm{B}, \theta_{u}$ reaches $\theta_{d}$ and the equilibrium is given by a zone at $\rho_{c}^{0}$ and by one at $\rho^{*}$.
TABLE I

SIMULATION PARAMETERS

\begin{tabular}{|c|c|c|c|}
\hline Parameter & Description & Value & Unit \\
\hline \hline$L$ & section length & 1 & $\mathrm{~km}$ \\
\hline$v$ & free-flow speed & 80 & $\mathrm{~km} / \mathrm{h}$ \\
\hline$w$ & congestion propagation speed & 20 & $\mathrm{~km} / \mathrm{h}$ \\
\hline$\rho_{M}$ & jam density & 250 & $\mathrm{veh} / \mathrm{km}$ \\
\hline$\rho^{*}$ & critical density & 50 & $\mathrm{veh} / \mathrm{km}$ \\
\hline$\varphi_{M}$ & maximum flow & 4000 & $\mathrm{veh} / \mathrm{h}$ \\
\hline$\rho_{f}^{0}$ & initial free-flow cell density & 30 & $\mathrm{veh} / \mathrm{km}$ \\
\hline$\rho_{c}^{0}$ & initial congested cell density & 150 & $\mathrm{veh} / \mathrm{km}$ \\
\hline$l_{0}$ & initial congestion length & {$[0.4 L, 0.4 L, 0.2 L]$} & $\mathrm{km}$ \\
\hline
\end{tabular}

\section{CONCLUSIONS}

A new Variable-Length cell transmission Model (VLM) has been presented in the paper as a basis to develop variable speed traffic control strategies. The proposed model differs from the standard Cell Transmission model since it consists of only three lumped state variables: two lumped densities and a third state variable describing the position of the congestion wave front. A modification of the model to capture also the rarefaction wave phenomenon is provided in the paper. Moreover, the major mathematical properties of the model are discussed. The paper also addresses the beneficial use of the new model in dealing with optimal speed control design. The method is illustrated on a closed ring road, where the model facilitates the determination of the free-speed such that the traveling time per turn is minimized.

\section{ACKNOWLEDGEMENTS}

Authors would like to acknowledge Tomas Pippia for helping with the simulations of this paper.

\section{REFERENCES}

[1] K. Aboudolas, M. Papageorgiou, and E. Kosmatopoulos. Store-andforward based methods for the signal control problem in largescalecongested urban road networks. Transportation Research Part C: Emerging Technologies, 17 (2). pp. 163-174, 2009.

[2] K. Aboudolas, M. Papageorgiou, A. Kouvelas, and E. Kosmatopoulos, A rolling-horizon quadratic-programming approach to the signal control problem in large-scale congested urban road networks. Transportation Research, Part C 17, 680-694, 2009.

[3] C. Canudas-de-Wit: Best-effort Highway Traffic Congestion Control via Variable Speed Limits, 50th IEEE Conference on Decision and Control and European Control Conference (IEEE CDC-ECC 2011), Orlando, Florida, Dec 2011.

[4] C. Canudas-de-Wit, F. Morbidi, L. Ojeda, A. Kibangou, I. Bellicot and P. Bellemain: Grenoble Traffic Lab: An Experimental Platform for Advanced Traffic Monitoring and Forecasting, IEEE Control Systems Magazine, Vol.35, pp.23-39, June 2015.

[5] C. F. Daganzo: The Cell Transmission Model: A Dynamic Representation of Highway Traffic Consistent with the Hydrodynamic Theory, Transportation Research Board, 28(4), 269-287, 1994.

[6] G. De Nunzio, C. Canudas-de-Wit, P. Moulin, Urban Traffic Ecodriving: A Macroscopic Steady-State Analysis, 2014 European Control Conference, Strasbourg, France, 2014.

[7] G. De Nunzio, C. Canudas-de-Wit, P. Moulin, Urban Traffic EcoDriving: Speed Advisory Tracking, 53rd IEEE Conference on Decision and Control, Los Angeles, CA, 2014.

[8] A. Ferrara, A. Nai Oleari, S. Sacone, S. Siri: Freeways as Systems of Systems: A Distributed Model Predictive Control Scheme, IEEE Systems Journal, Vol. 9, pp. 312-323, 2015.

[9] A. Ferrara, S. Sacone, S. Siri: Event-triggered model predictive schemes for freeway traffic control, Transportation Research C, Vol. 58, pp. 554?567, 2015. 

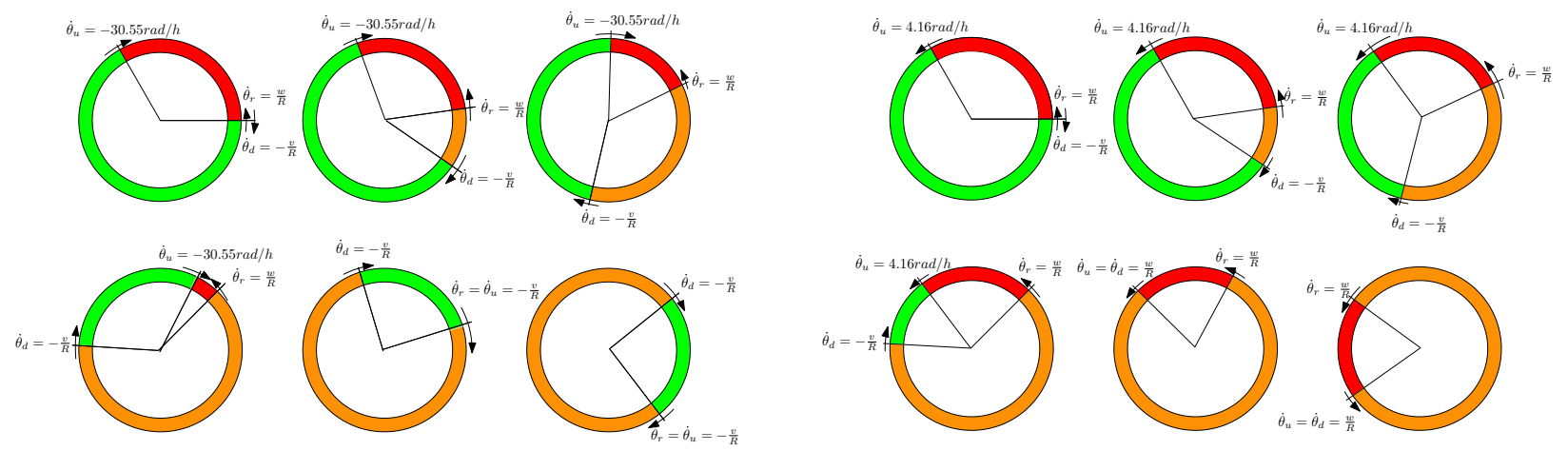

Fig. 6. Snapshots of time evolution of the ring road traffic: left figures show the results in case A, whereas right figures those in case B. Free-flow zones are indicated in green, critical-density zones in orange and congested zones in red.

[10] D. C. Gazis and R. B. Potts. The oversaturated intersection, 2nd International Symposium on Traffic Theory. London, UK, pp. 221237, 1963

[11] S. Godunov: A difference scheme for numerical solution of discontinuous solution of hydrodynamic equations, Matematicheskii Sbornik, Vol. 47, pp. 271-306, 1959.

[12] G. Gomes, R. Horowitz, A. A. Kurzhanskiy, J. Kwon, and P. Varaiya: Behavior of the Cell Transmission Model and Effectiveness of Ramp Metering, Transportation Research C, Vol. 16, pp. 485-513, 2008.

[13] R. Horowitz, A. May, A. Skabardonis, P. Varaiya, M. Zhang, G. Gomes, L. Muñoz, X. Sun and D. Sun: Design, field implementation and evaluation of adaptive ramp metering algorithms. California Partners for Advanced Transit and Highways (PATH), 2005.

[14] D. Jacquet, C. Canudas De Wit and D. Koenig: Optimal Control of Systems of Conservation Laws and Application to Non-Equilibrium Traffic Control, 13th IFAC Workshop on Control Applications of Optimization, Paris, France 2006.

[15] D. Jacquet, J. Jaglin, D. Koenig and C. Canudas De Wit: Non-Local Feedback Ramp Metering Controller Design, 11th IFAC Symposium on Control in Transportation Systems (CTS), Delft, The Netherlands, 2006.

[16] M. J. Lighthill and G. B. Whitham: On kinematic waves II: A theory of traffic flow on long crowded roads, Proceedings of the Royal Society of London. Series A, Mathematical and Physical Sciences, Vol. 229, pp. 317-345, 1955.

[17] X.-Y. Lu, P. Varaiya, R. Horowitz, D. Su, and S. Shladover: A new approach for combined freeway variable speed limits and coordinated ramp metering, 13th International IEEE Conference on Intelligent Transportation Systems. Madeira Island, Portugal, 2010.

[18] M. Papageorgiou: Freeway ramp metering: an overview, IEEE Transactions on Intelligent Transportation Systems, Vol. 3, No.4, pp. 271-281, 2002.

[19] P. I. Richards: Shock Waves on the Highway, Operations Research, Vol. 4, pp. 42-51, 1956.

[20] A. Smadi, J. Baker and S. Birst: Advantages of Using Innovative Traffic Data Collection Technologies. Applications of Advanced Technology in Transportation, ASCE Library, Chapter 102, pp. 641-646, 2006.

[21] M. Treiber and A. Kesting: Traffic flow dynamics - Data, models and simulation, Springer, 2013.

[22] I. Yperman, The link Transmission Model for Dynamic Network Laoding, Ph.D, Katholieke Universiteit Leuven ATHOLIEKE UNIVERSITEIT LEUVEN, June 2007.

[23] P. Varaiya. Max pressure control of a network of signalized intersections, Transportation Research Part C Vol.36, (2013), 177-195. 